\title{
Neutrons production on the IPHI accelerator for the validation of the design of the compact neutron source SONATE.
}

\author{
Hoang Ngoc $\operatorname{Tran}^{1}$, Frédéric Ott ${ }^{2}$, Jacques Darpentigny ${ }^{2}$, Anthony Marchix ${ }^{1}$, Alain Letourneau ${ }^{1}$, Nicolas Chauvin ${ }^{1}$, Fabien \\ Prunes $^{2}$, Benoît Homatter ${ }^{2}$, Burkhard Annighöfer ${ }^{2}$, Alain Menelle ${ }^{2}$, Jérôme Schwindling ${ }^{1}$ \\ ${ }^{1}$ IRFU, CEA, Université Paris-Saclay, F-91191 Gif-sur-Yvette, France \\ ${ }^{2}$ LLB, CEA-CNRS, Université Paris-Saclay, F-91191 Gif-sur-Yvette, France
}

\begin{abstract}
We aim at building a compact accelerator-based neutron source (CANS) which would provide a thermal neutron flux on the order of $4 \times 10^{12} \mathrm{n} \cdot \mathrm{s}^{-1} \cdot \mathrm{cm}^{-2} \cdot \mathrm{sr}^{-1}$. Such a brilliance would put compact neutron sources on par with existing medium flux neutron research reactors for neutron scattering experiments. We performed the first neutron production tests on the IPHI proton accelerator at Saclay at a proton energy of $3 \mathrm{MeV}$. The thermal neutron flux were measured using gold foil activation and ${ }^{3} \mathrm{He}$ detectors. The measured flux were compared with GEANT4 Monte Carlo simulations (10.4) in which the whole experimental setup was modelled. There is a good agreement between the experimental measurements and the Monte-Carlo simulations. The available modelling tools will allow us to optimize the whole Target Moderator Reflector assembly together with the neutron scattering spectrometer geometries for the design of the neutron scattering facility SONATE.
\end{abstract}

\section{Introduction}

There is currently an interest in developing compact neutron sources based on low energy proton accelerators (10-100 MeV) [1]. Such sources could serve as neutron sources for neutron scattering to replace small ageing nuclear reactors [2] or in hospitals for Boron Neutron Capture Therapy [3-4]. There are already several such projects on-going around the world. They are generally referred to as CANS, Compact Accelerator-based Neutron Sources. The currently most advanced is LENS Low Energy Neutron Source at Indiana University [5]. The CANS operating or under construction have gathered into the UCANS, Union for Compact Accelerator-driven Neutron Sources [6].

At Saclay we are considering a similar possibility to replace the Orphée research reactor. Our final goal is to build a higher end facility SONATE which could serve a suite of about 10 neutron scattering instruments. The facility would operate at a proton energy around $20 \mathrm{MeV}$, in pulsed mode to use time-of-flight techniques with a typical power on the target of $80 \mathrm{~kW}$.

Our first aim has been to experimentally validate the neutron production and moderation obtained by Monte Carlo simulations using GEANT4. Once reliable simulation tools are available we shall be able to reliably estimate the performances of a CANS for neutron scattering experiments (from the source to the spectrometers) and compare its performances to existing facilities (reactor or spallation based).
As a starting point we are using the IPHI proton accelerator at Saclay which is able to produce high current proton beams (up to $90 \mathrm{~mA}$ ) [7] with proton energies of $3 \mathrm{MeV}$.

The Monte Carlo simulation toolkit GEANT4 is a library for the simulation of the transport of particles through matter. This tool is widely used in various applications from high-energy to very low-energy physics, including astrophysics and space science, medical physics and radiation protection [8]. Within the neutron physics package of GEANT4, the particlehp (high precision) option has to be used for neutron energies below $20 \mathrm{MeV}$ as it refers to the library G4NDL that is based on the evaluated nuclear data library ENDF/B-VII.0 [9]. Moreover, this latter includes the thermal scattering libraries that take into account the molecular effects occurring for neutron energies below $4 \mathrm{eV}$ when considering moderator and reflector materials such as water, polyethylene, etc. Recently, the validation of the neutron transport treatment in GEANT4-10.3 for the thermal energy range was performed by inter-comparison with the standard Monte Carlo code MCNP-6.1 on microscopic and macroscopic observables [10]. It was pointed out some sampling issues for the data treatment of the thermal scattering libraries included in the G4NDL for the versions of GEANT4-10.4. These improvements concern the treatment of nuclear interactions involved during the propagation of neutrons in a complex geometry, in particular the moderation process. But, to ensure the reliability of simulation tools, the neutrons production has to be validated as the thermal neutron

\footnotetext{
* Corresponding author: frederic.ott@ecea.fr
} 
distributions within the moderator are sensitive to the double differential cross sections in energy and angle of primary neutrons.

Within this framework, we have performed experiments in order to measure the primary neutron source characteristics associated to the reaction ${ }^{9} \mathrm{Be}(\mathrm{p}, \mathrm{n})$, as well as the thermal neutron flux in various locations by using a simplified polyethylene (PE) moderator. This paper describes the experimental and simulation methods that was used to measure the thermal neutron flux inside and outside the moderator, including the thermal neutron spectra and the neutron beam divergences, as well as the fast-neutron angular distribution related to the reaction ${ }^{9} \mathrm{Be}(\mathrm{p}, \mathrm{n})$. The method for calibrating the ${ }^{3} \mathrm{He}$ detector efficiency via wavelength is also discussed.

\section{Experimental setup}

\subsection{Proton source}

The experiments have been performed on the IPHI proton accelerator which is based at CEA Saclay, France. The accelerator consists of a proton source (SILHI) of energy $95 \mathrm{keV}$, a Low Energy Beam Transport Line and a Radio Frequency Quadrupole to accelerate the protons to an energy of $3 \mathrm{MeV}$. The accelerator is designed to operate in continuous mode with proton currents up to $100 \mathrm{~mA}$ which corresponds to a total power of $300 \mathrm{~kW}$. For the current experiments dedicated to validations of neutron production and moderator Monte Carlo simulations we have operated the accelerator at a very low power of about $10 \mathrm{~W}$, both to avoid any target damage and for radioprotection issues. The accelerator was operated in pulsed mode with proton pulses of length $100 \mu$ s and with a repetition rate of $1 \mathrm{~Hz}$ and a peak current of $30 \mathrm{~mA}$. Rather narrow thermal neutron pulses are thus obtained so that precise time-of-flight measurements can be performed.

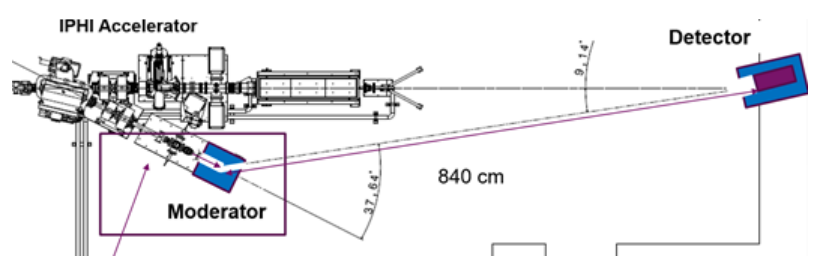

Fig. 1. Sketch of the experimental set-up. The target is installed on a deviator line and the neutron detectors are set at $8.4 \mathrm{~m}$ from the target so as to be able to perform time-of-flight measurements.

\subsection{Target and moderator assembly}

In order to produce neutrons we opted for a beryllium target of thickness $0.5 \mathrm{~mm}(99.0 \%)$ which stopped all incident protons. The target was attached on an aluminium support with titanium screws in order to minimize neutron activation. This support was air cooled. The proton beam size was limited to $16 \mathrm{~mm}$ in diameter. The proton current incident on the target was continuously measured so as to have a precise value of the incident particle flux and be able to precisely estimate the neutron production. An electron repelling electrode set at a potential of $200 \mathrm{~V}$ was set in front of the beryllium target so as to prevent any bias in the target current measurement. The target was installed in a polyethylene (PE) moderator box $\left(300 \times 300 \times 400 \mathrm{~mm}^{3}\right)$ so as to cool down the neutron to thermal energies (around $26 \mathrm{meV}$ ). A $20 \mathrm{~mm}$ diameter hole was drilled through the moderator from the position where the thermal neutron density was expected to be the highest to the outside of the PE box (see Fig. 2).

In order to change the moderator geometry, it was possible to insert PE "plugs" inside the exit hole so as to fill more or less the neutron extraction channel.

The whole experimental setup (accelerator - target moderator - detectors) was installed in a $2 \mathrm{~m}$ thick concrete casemate.

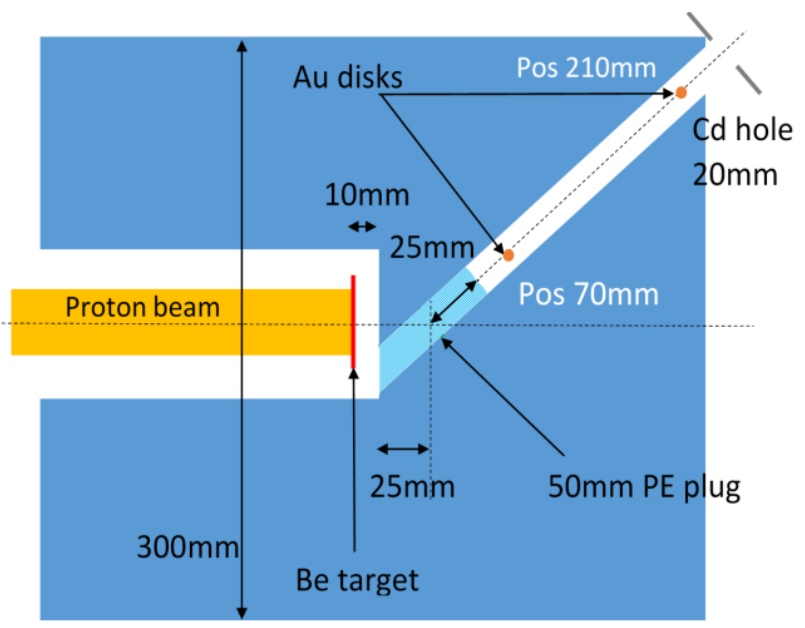

Fig. 2. Target and moderator assembly

\subsection{Neutron detection}

\subsubsection{Fast neutron detection}

A technical difficulty arises from the fact that since we operate in pulsed mode ( $100 \mu$ s proton pulses width), the instantaneous neutron flux is very high. This prevented using a regular Bonner sphere. A dedicated acquisition chain was mounted consisting of a very small ${ }^{3} \mathrm{He}$ detector ( $1 \mathrm{~cm}$ in diameter) encased in a cubic polyethylene setup. We made sure that even though we operated in pulse mode we did not have events pile-up in the ${ }^{3} \mathrm{He}$ detector. This led to acquisition times on the order of 5-10 minutes to obtain a good statistics in the measurement of the fast neutrons around the source.

\subsubsection{Thermal neutron detection}

The thermal neutron flux was measured at $8.4 \mathrm{~m}$ from the source using time-of-flight mode and a ${ }^{3} \mathrm{He}$ detector. The ${ }^{3} \mathrm{He}$ detector was surrounded with a shielding made of polyethylene, as shown in

Fig. 3, and the detector was placed at $20 \mathrm{~cm}$ from the aperture of $6 \mathrm{~cm}$ in diameter. The solid angle covered by the detector was thus reduced to about $8^{\circ}$ allowing to reduce the background noise from neutrons scattered and 
moderated on the concrete walls of the casemate. Moreover, a layer of $\mathrm{B}_{4} \mathrm{C}$ was installed around the ${ }^{3} \mathrm{He}$ detector to capture the thermal neutrons coming from the polyethylene shielding.

Even though the source was operated at a modest $10 \mathrm{~W}$ power we were very close to the saturation limit of regular ${ }^{3} \mathrm{He}$ tubes $(\sim 20 \mathrm{kHz} /$ tube $)$ so that the detection area was limited to $20 \mathrm{~cm}^{2}$, corresponding to the front face of a single 2 inches ${ }^{3} \mathrm{He}$ detector tube (NH 50).

Thermal neutrons $(1.8 \AA)$ travel at a speed of $2200 \mathrm{~m} / \mathrm{s}$ so that the travel time over $8.4 \mathrm{~m}$ is $3.8 \mathrm{~ms}$. For a proton pulse width of $100 \mu \mathrm{s}$, the energy resolution is thus on the order $0.1 / 3.8=2.6 \%$ which is good enough to measure a rather precise neutron energy distribution. Note that besides the proton pulse length, the moderation process leads to an intrinsic neutron pulse broadening which reduces the energy resolution. Monte-Carlo simulations provide an estimate of a broadening on the order of $100-150 \mu \mathrm{s}$ in a PE moderator [11-12].

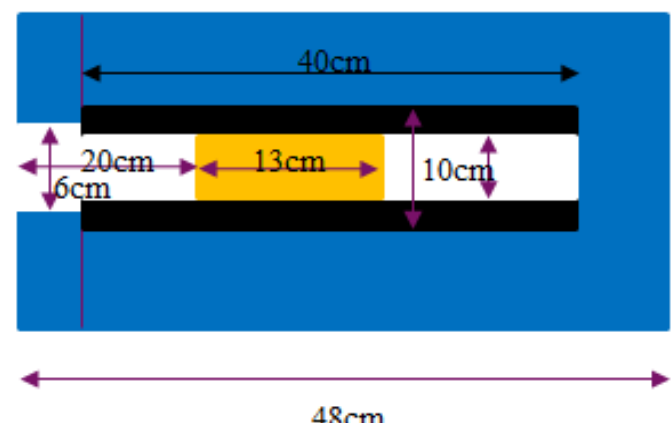

Fig. 3. Polyethylene (blue) moderator (left). ${ }^{3} \mathrm{He}$ detector system design (right).

The ${ }^{3} \mathrm{He}$ detector used in horizontal position suffered from an issue related to a dead zone at the front of the detector where neutrons are absorbed in the ${ }^{3} \mathrm{He}$ gas but not detected due to the absence of an amplification electric field at top end of the detector [13]. This dead zone bias the detection efficiency as a function of the wavelength. Short wavelengths penetrate deeply in the detector and are thus almost all detected while a large fraction of long wavelengths $(>3 \AA)$ are absorbed in the front part of the ${ }^{3} \mathrm{He}$ gas but not detected. The "dead zone" of the detector was mapped with a pencil beam on the PRISM spectrometer at Orphée at a wavelength of $4 \AA$ (see Fig. $4 \mathrm{a})$. The dead zone extends over $26 \mathrm{~mm}$ from the top of the detector. The detector efficiency is deduced by integrating the ratio between the effective distribution and the ideal distribution to the detector length. Fig. $4 \mathrm{~b}$ is provided the calculated detector efficiency as function of the neutron energy (via TOF). The measurement have been performed with a spectrometer of the Orphée reactor for a neutron beam of 4.3 ångström that confirms the calculated detector efficiency.
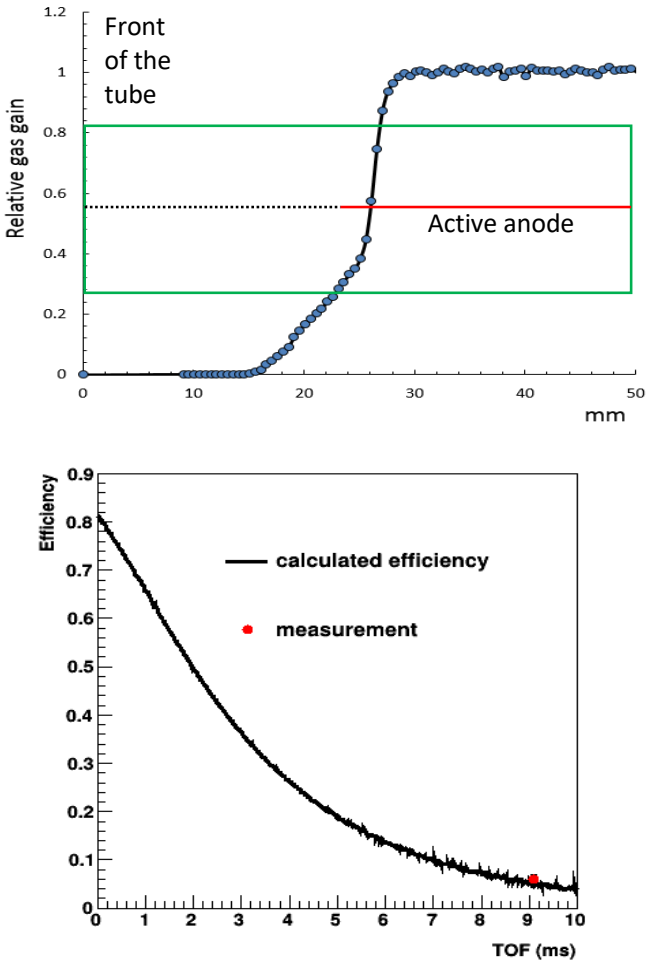

Fig. 4. (a) Detection efficiency along the length of the ${ }^{3} \mathrm{He}$ tube. The dead zone is approximately $26 \mathrm{~mm}$ long; (b) Calculated detector efficiency with GEANT4 together with a superimposed experimental calibration point (red) measured on Orphée. The average detection efficiency of the detector was $30 \%$.

\subsubsection{Gold foil activation}

In order to validate the Monte-Carlo simulations inside the moderators, gold disks were positioned at various positions inside the moderator. Disks with a diameter of $6 \mathrm{~mm}$ and thickness $200 \mu \mathrm{m}$ were used. The weight of these disks was $112 \mathrm{mg}$ so that even when operating at $10 \mathrm{~W}$, measurable activation of the gold was achieved within 15-30 minutes of operation. Bare disks and disks enclosed in Cadmium were measured so as to be able to estimate the thermal and fast neutron flux [14]. The Cd absorption cut-off edge is at around $500 \mathrm{meV}(\sigma=100$ barn) so that the bare disks are activated by the whole neutron spectrum irradiation and the $\mathrm{Cd}$ covered disks are only activated by the fast neutrons. These gold activation measurements provide a simple and quantitative way of measuring the thermal neutron flux. However, while the measurement of the gold activation is quantitative and reliable, in order to calculate an absolute neutron flux, an a priori knowledge of the neutron energy distribution is necessary. Hence either an assumption of neutron energy distribution has to be made (Maxwellian distribution centered around $26 \mathrm{meV}$ for example) or the activation must be calculated using a simulated neutron energy distribution. Four gold disks with same size (with and without $\mathrm{Cd}$ casing) have been put into the moderator at positions 1 and 2 (see Fig. 2) at $70 \mathrm{~mm}$ and $210 \mathrm{~mm}$ from the intersection of the proton beam axis with the neutron extraction hole. Their activation has been calculated during the Monte Carlo simulations. The measured 
activation values have then been compared with the calculated activation values.

\section{Experimental results}

\subsection{Fast neutron yield and angular distribution}

In order to use a reliable model for the fast neutron term an accurate knowledge of the fast neutron emission rate and angular distribution is required. Moreover, no data are available for $3 \mathrm{MeV}$ protons, while they are needed for validating the models implemented in GEANT4 in the framework of the prototype tests at $3 \mathrm{MeV}$ protons. In consequence, we performed a new measurement of the angular distribution of the fast neutrons produced by a 3 $\mathrm{MeV}$ proton beam impinging on a thick beryllium target.

The angular distribution of neutrons related to the reaction ${ }^{9} \mathrm{Be}(\mathrm{p}, \mathrm{n})$ was measured by using a $\mathrm{PE}-{ }^{3} \mathrm{He}$ detector system that consists of $10 \mathrm{~cm}$ thick PE in front of a ${ }^{3} \mathrm{He}$ counter (4.5 mm radius, $200 \mathrm{~mm}$ length). The aim of the polyethylene was to moderate part of incoming neutrons aiming to be captured in the ${ }^{3} \mathrm{He}$ detector. The $\mathrm{PE}-{ }^{3} \mathrm{He}$ detector was located at a distance of $2 \mathrm{~m}$ from the target. The detector was shielded from parasitic thermal neutron scattered from the concrete wall with a $\mathrm{Cd}$ sheet. The Proton beam intensity was limited to $2.2 \mu \mathrm{A}$ to avoid pileup effects in the ${ }^{3} \mathrm{He}$ counter. The fast neutron yield was measured for six angles around the target, from 0 to $150^{\circ}$ by steps of $30^{\circ}$.

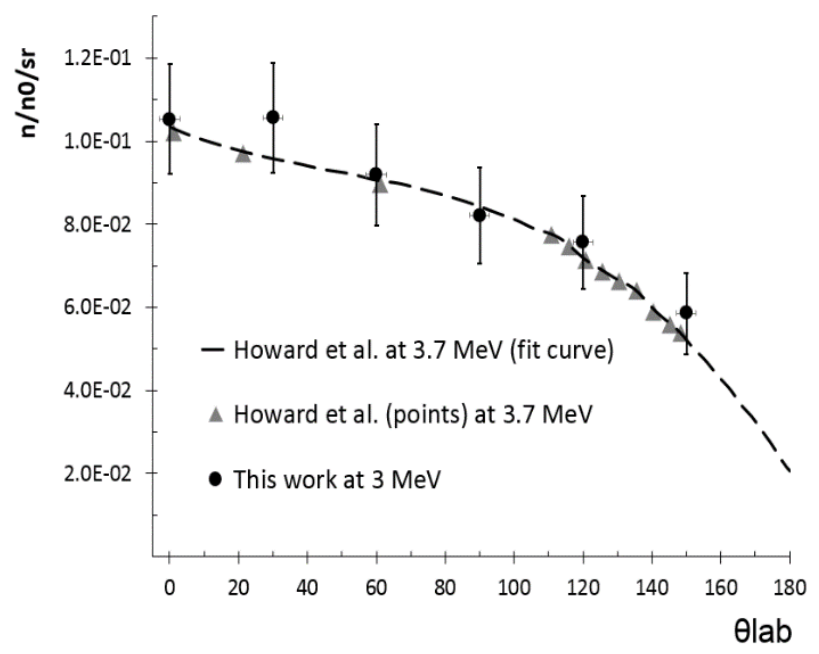

Fig. 5. Angular distributions of the neutrons from the ${ }^{9} \mathrm{Be}(\mathrm{p}, \mathrm{n})$ reaction of $3 \mathrm{MeV}$ proton beam compared with the results of Howard et al. [15] at 3.7 MeV. The angular resolution was $\pm 2.8^{\circ}$.

Fig. 5 shows the measured angular distributions of neutrons produced in the reaction ${ }^{9} \mathrm{Be}(\mathrm{p}, \mathrm{xn})$ for $3 \mathrm{MeV}$ protons. Our new measurements confirms those from Howard et al. [15] performed with a proton beam of 3.7 $\mathrm{MeV}$. The comparison is normalized to the neutron rate of each measurement (n0) which is adjusted by Howard's measurements [12]. The data were modelled with a fourth order polynomial to build the fast-neutron source used in the GEANT4 simulations.

\subsection{Thermal neutron yield}

\subsubsection{Gold foil activation inside the moderator}

Table 1 provides the data values obtained on the activity of the different gold foils. All the details have been fully described in Allen et al. [17]. The gold disks are irradiated for durations ranging from 15 to 22 minutes which lead to measurable gamma activity from ${ }^{198} \mathrm{Au}$. It can be remarked that the Cd-layer strongly decreases the activity of each gold foils, especially for the furthest location $(210 \mathrm{~mm})$ that is below the detection limit. The measured activities of the gold foils have $\sim 23 \%$ of incertitude due to determination of gold foils activation. Note that the measurements were performed by considering a $50 \mathrm{~mm}$ thick PE plug in the neutron extraction channel (see Fig. 2). The thermal neutron flux (showed in Table 1) associated to these measurements have been deduced based on the cross section of data library ENDF/B-VII.0.

\begin{tabular}{|c|c|c|c|c|c|}
\hline $\begin{array}{c}\text { Gold } \\
\text { activation }\end{array}$ & $\begin{array}{c}\text { Curr } \\
\text { ent } \\
(\mu \mathrm{C})\end{array}$ & $\begin{array}{c}\text { Irradiat } \\
\text { ion time } \\
\text { (s) }\end{array}$ & $\begin{array}{c}\text { Activation } \\
(\mathbf{B q} / \mathrm{g})\end{array}$ & $\begin{array}{c}\text { Thermal } \\
\text { neutron flux } \\
(<0.4 \mathrm{eV}) \\
\left(\mathrm{n} / \mathrm{cm}^{2} / \mu \mathrm{C}\right)\end{array}$ & $\begin{array}{l}\text { Systema } \\
\text { tic } \\
\text { errors }\end{array}$ \\
\hline $\begin{array}{c}\text { Foil } 1 \\
\text { without } \\
\text { Cd }\end{array}$ & 2.9 & 900 & 2700 & $1.21 \times 10^{6}$ & \\
\hline $\begin{array}{c}\text { Foil } 1 \\
\text { with Cd }\end{array}$ & & & 533 & & $\sim 23 \%$ \\
\hline $\begin{array}{c}\text { Foil } 2 \\
\text { without } \\
\text { Cd }\end{array}$ & 2.6 & 1380 & 57 & $1.70 \times 10^{4}$ & \\
\hline $\begin{array}{c}\text { Foil } 2 \\
\text { with Cd }\end{array}$ & & & $<9.7$ & & \\
\hline
\end{tabular}

Table 1. The measured activation foils were used to reduce thermal neutron flux (less than $0.4 \mathrm{eV}$ ).

\subsubsection{Thermal beam mapping}

Outside the moderator, the thermal neutron flux was mapped with a ${ }^{3} \mathrm{He}$ detector by scanning the detector position in the $\mathrm{X}$ and $\mathrm{Y}$ directions to ensure we were aiming at the peak flux. Fig. 6 presents the thermal neutron beam divergences in both $\mathrm{X}$ and $\mathrm{Y}$ axis at the distance $8.4 \mathrm{~m}$. We observe a fair agreement between the experimental data (red dots) and the calculation (dark line). The systematic underestimate between the experimental and calculated data may originate from secondary thermal neutron emission background in the experimental data. 

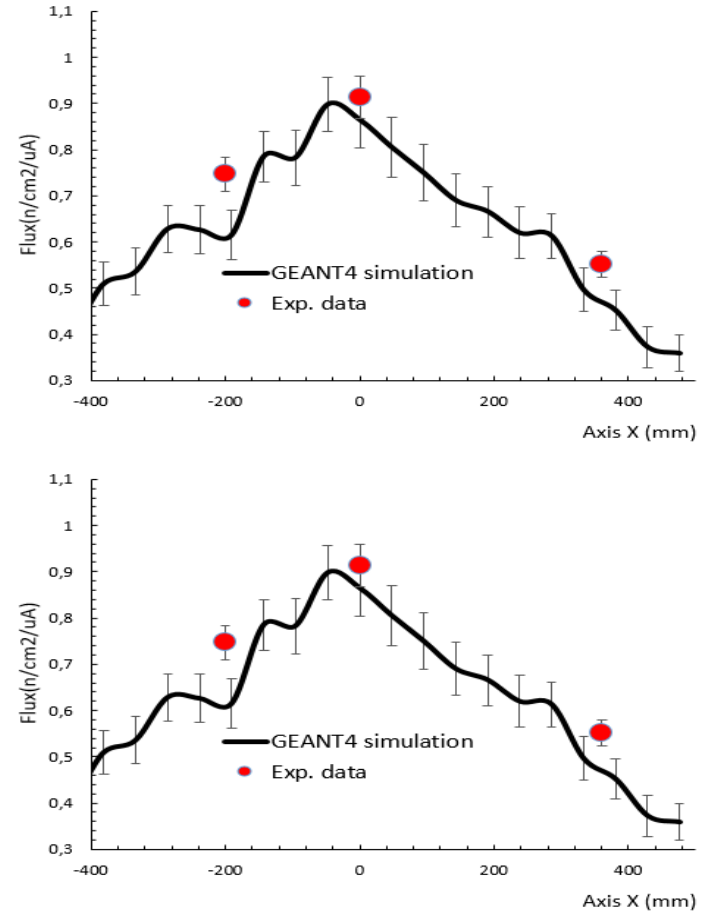

Fig. 6. Thermal neutron beam divergences at $8.4 \mathrm{~m}$ from the target. The positive direction of Y axis is in the proton beam's side. The origin is at $8.4 \mathrm{~m}$.

Fig. 7 summarizes the thermal neutron flux measurements obtained from gold foil activation and time of flight measurement along the neutron flight path (red dots). The result of the simulation performed with GEANT4 is also provided. A very good agreement is obtained between the measurements and the simulation result. In this simplified moderator geometry, it is possible to obtain a thermal neutron flux of about $1000 \mathrm{~cm}^{-2} \cdot \mathrm{s}^{-1}$ at the exit of the moderator.

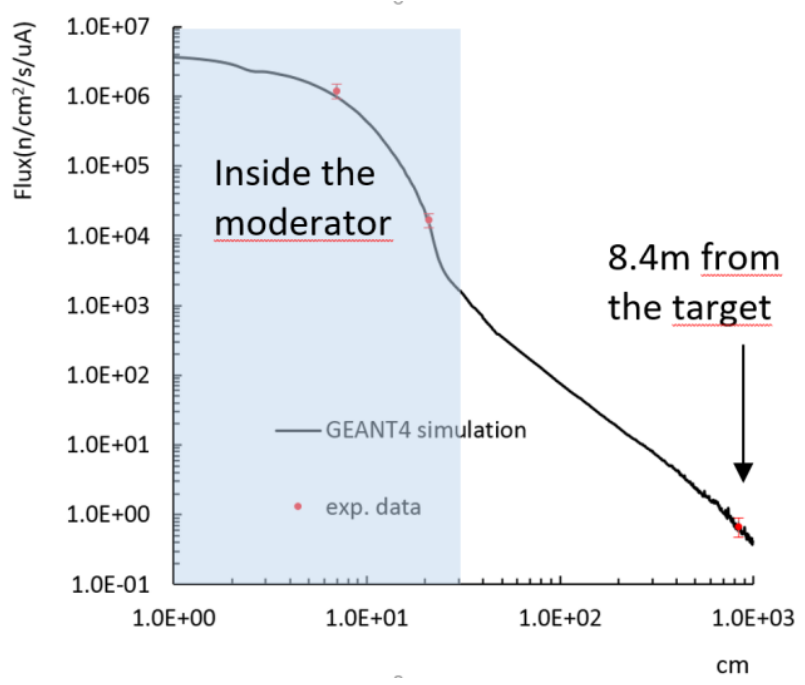

Fig. 7. Thermal neutron flux (less than $0.4 \mathrm{eV}$ ) as function of the distance between the target and $3 \mathrm{He}$ counter given by Geant 4 simulation and compared with measurements. Measured flux inside the moderator using the gold foil activation technics and outside the moderator using ${ }^{3} \mathrm{He}$ counter.

\subsubsection{Thermal neutron energy spectrum}

The comparison of the neutron time-of-flight spectrum requires a high statistic for the simulations, and consequently a high computing time, as the number of neutron events reaching the ${ }^{3} \mathrm{He}$ detector at $8.4 \mathrm{~m}$ is extremely low in the simulation. To reduce the computational time, the GEANT4 simulation has been split into two steps:

- The first step: $10^{9}$ neutrons were generated at the target position according to the measured angular distribution discussed above. This simulation performs the neutron transport within the polyethylene moderator. The neutron spectrum is stored at the entrance of the ${ }^{3} \mathrm{He}$ detector, as well as the neutron angular distribution and the neutron position in the transversal plane.

- The second step: based on the neutron distributions in energy, in angle and in position obtained from the first step, we generated $10^{7}$ neutrons from the surface in front of the ${ }^{3} \mathrm{He}$ detector. The ${ }^{3} \mathrm{He}(\mathrm{n}, \mathrm{p})$ reactions are tracked in the detector volume and the energy distribution of incident neutrons is recorded.

Considering the time of the moderation processes (less than $2 \%$ in average) is negligible in comparison with the TOF in the large distance from the moderator to the detector $(8.4 \mathrm{~m})$, we convert the neutron energy spectrums to the travel time. The amount of attenuation due to air is about $\sim 8 \%$ for the distance of $8.4 \mathrm{~m}$. Fig. 8 . shows the comparison of the neutron TOF spectrum obtained for the $50 \mathrm{~mm}$ thick PE plug configuration (see the geometry in

Fig. 3a) between simulations and experimental data. A general agreement in the thermal Maxwell distribution with the measurement is showed in Fig. 7. An underestimation of simulation below $1 \mathrm{~ms}$ is due to the gamma background of the counter. Note that the calculation is corrected by the detector efficiency and the measurement is also corrected by the measured thermal neutron background in the casemate (figure 3 ).

The time-of-flight (ToF) measurements were performed by setting a single ${ }^{3} \mathrm{He}$ detector at a distance of $8.4 \mathrm{~m}$ in the direction of the extraction hole. The incident neutron flux was measured with time channels of width $20 \mu$ s. The start of the acquisition was synchronized with the clock signal of the accelerator radio-frequency. Each ToF spectrum was acquired in 5 to 10 minutes. Figure 8 shows examples of ToF spectra measured in various conditions so that the contributions of various types of particles could be identified and quantified. The blue curve corresponds to the "reference signal" as measured with the box described on

Fig. 3. The peak in the region between 1 and $6 \mathrm{~ms}$ corresponds to the thermal neutrons. The thermal spectrum is peaked around $\mathrm{t}=2.2 \mathrm{~ms}$ corresponding to a neutron speed of $3800 \mathrm{~m} / \mathrm{s}$, i.e. $\lambda=1.05 \AA$. The neutron spectrum can be fitted with a Maxwellian distribution centred on a temperature of $350 \mathrm{~K}$. While this is higher 
than the actual moderator temperature, it is no surprise as neutron moderated with hydrogenated materials are always under-moderated contrary to thermal neutron produced in a $\mathrm{D}_{2} \mathrm{O}$ reactor vessel where the interaction time is much longer.

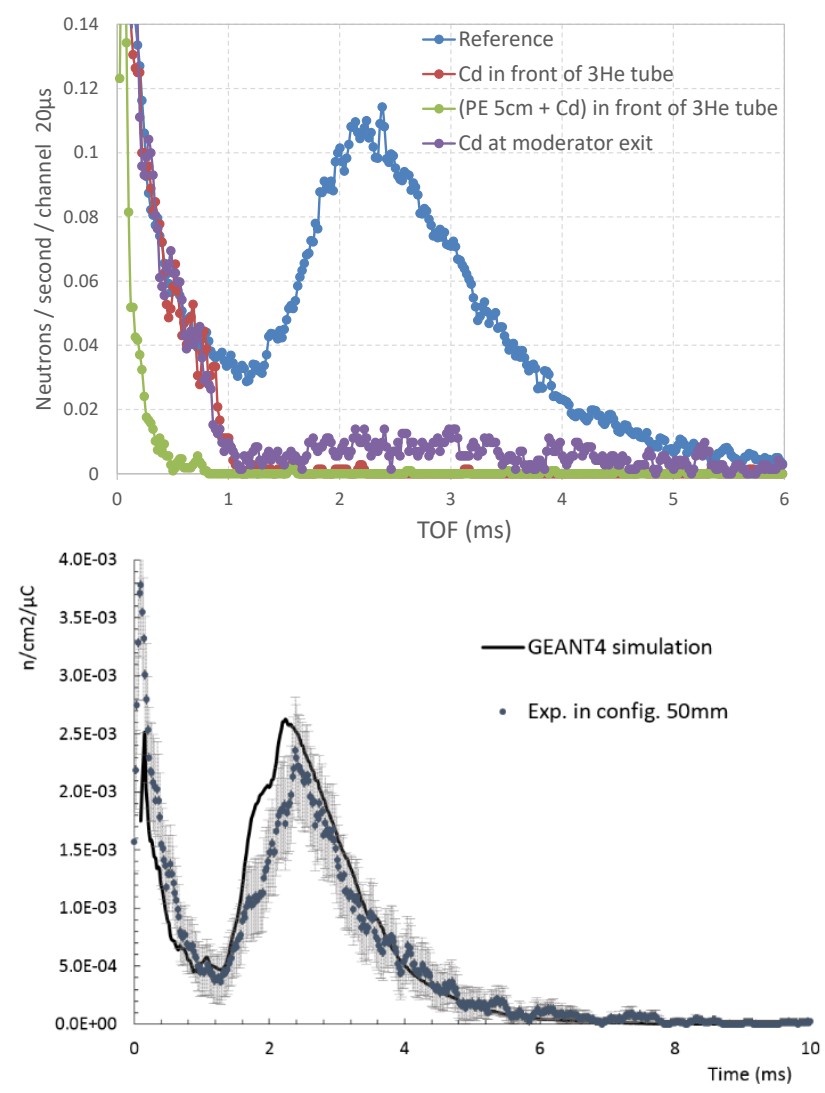

Fig. 8. The comparison of the ToF spectrum between experimental data and Geant 4 calculation.

Extra contributions are observed below $\mathrm{TOF}<1 \mathrm{~ms}$. In order to identify these contributions, a Cadmium sheet was put in front of the detector shielding box (Figure 8a, red dots). Above $\mathrm{t}=1 \mathrm{~ms}$, there is no residual signal. $\mathrm{t}=1 \mathrm{~ms}$ corresponds to a neutron energy of $0.5 \mathrm{eV}$ which is the neutron Cd cut-off. Hence it is demonstrated that the

1. C. Andreani, C-K. Loong and G. Prete, Eur. Phys. J. Plus 131 (2016) 217. DOI: 10.1140/epjp/i2016-16217-1

2. U. Rücker, T. Cronert, J. Voigt, et al, Eur. Phys. J. Plus 131 (2016) 19. DOI 10.1140/epjp/i2016-16019-5

3. A. J. Kreiner, M. Baldo, J.R. Bergueiro, D. Cartelli, et al., Appl. Radiat. Isot. 88 (2014) 185-189.

4. H. Kumada, A. Matsumura, H. Sakurai et al, Appl. Radiat. Isot. 88 (2014) 211-215.

5. C.M. Lavelle et al, NIM A 587 (2008) 324-341

6. UCANS, www.ucans.org/facilities.html

7. R. Gobin et al., Rev. Sci. Instrum. 73 (2002) pp 922.

8. J. Allison, et al., Nucl. Instr. and Meth. A, 835 (2016) 186-

225.

9. M.B. Chadwick et al., Nucl. Data Sheets 107 (2006) 2931.

10. H.N. Tran et al., Nuclear Inst. and Methods in Physics

Research A 893 (2018) 84-94 measured spectrum actually corresponds only to thermal neutrons. In a third measurement, thermal neutrons were prevented from exiting the moderator with a $\mathrm{Cd}$ sheet at the moderator exit; fast neutron could still escape the moderator. The measured signal (purple dots) shows that a residual neutron signal is then observed for $\mathrm{t}>1 \mathrm{~ms}$. It corresponds to the fast neutrons moderated into the casemate walls and eventually arriving into the detector. This allows telling that $85 \%$ of the measured thermal neutrons are actually the ones coming from the moderator extraction hole while $15 \%$ of the neutron are secondary emission thermal neutron originating from the moderation of fats neutron in the casemate wall. In further measurements, the collimation system should be improved. In a $4^{\text {th }}$ measurement the fast neutron contribution was stopped with a PE block followed by a $\mathrm{Cd}$ sheet in front of the detector shielding entrance. The data (green dots) shows that there is a remnant short time contribution of either epithermal neutrons or gammas. These different contributions can easily be discriminated using the ToF measurements. However, due to the finite proton pulse length $(100 \mu \mathrm{s})$, the finite moderation time $(100 \mu \mathrm{s})$ and the short flight path, no quantitative information can be retrieved for neutron energies above $30 \mathrm{eV}$ since above this energy, all ToF information is blurred in the first few time channels.

\section{Conclusion}

This paper describes the experimental and simulation methods to determine angular distribution of primary fast neutrons of ${ }^{9} \mathrm{Be}(\mathrm{p}, \mathrm{n})$ and the thermal neutron flux inside and outside the PE moderator including thermal neutron energy spectra and neutron beam divergences based on $3 \mathrm{MeV}$ proton beam of IPHI accelerator. The efficiency of the used ${ }^{3} \mathrm{He}$ detector for these measurements is estimated. Good agreements between GEANT4 simulation and measurement data are observed. This gives us confidence in the reliability of the GEANT4 for the quantitative description of neutron moderators.

\section{References}

11. Lavelle CM et al, Nucl Instrum Methods A 587 (2008) 324-3410

12. B. Zhong, T.J. Liang, Q.X. Feng, G.L. Yu, K. Wang, C.-K. Loong, Physics Procedia 26 (2012) $25-30$.

13. K. Kudo et al. Nuclear Instruments and Methods in Physics Research B 213 (2004) 305-309.

14. D.A. Allen et al., Nuclear Instruments and Methods in Physics Research A 353 (1994) 128-133.

15. W.B. Howard and J.C Yanch, Nuc. Sci. and Eng. 138 (2001) 145 . 